\title{
Numerical study of time-dependent ferrofluid flow past a cylinder in the presence of stationary magnetic field
}

\author{
Anupam Bhandari ${ }^{1}$ iD
}

Received: 6 August 2020 / Accepted: 22 December 2020 / Published online: 12 January 2021

(c) The Author(s) 2021 OPEN

\begin{abstract}
This work investigates time-dependent ferrofluid flow past in a cylinder in the presence of a 10 kilo-ampere per meter magnetic field. The Reynolds number is about a hundred to keep the laminar flow and it is high enough to form a von Karman vortex street. This study presents the results for the velocity distributions, pressure distributions, lift coefficient, and drag coefficient under the influence of the stationary magnetic field. These results are compared with the flow in the absence of the magnetic field. The presence of the magnetic field diminishes the velocity distributions in the flow due to magnetization force and magnetic field dependent viscosity. This reduction in the velocity reduces the average velocity in the flow and therefore the magnetic field intensity enhances the coefficients of drag and lift. In the presence of the applied magnetic field, the velocity drops from 2.19 to $1.97 \mathrm{~m} / \mathrm{s}$ at $\mathrm{t}=7 \mathrm{~s}$. However, the lift coefficients enhance from $3 \mathrm{~m}^{2} \mathrm{~s}^{2} / \mathrm{kg}$ to $3.4 \mathrm{~m}^{2} \mathrm{~s}^{2} / \mathrm{kg}$ and the drag coefficient enhances from 0.9 to $3 \mathrm{~m}^{2} \mathrm{~s}^{2} / \mathrm{kg}$. The numerical simulation of the problem is obtained using the finite element method in COMSOL Multiphysics.
\end{abstract}

Keywords Ferrofluid · Cylindrical flow · Magnetic field
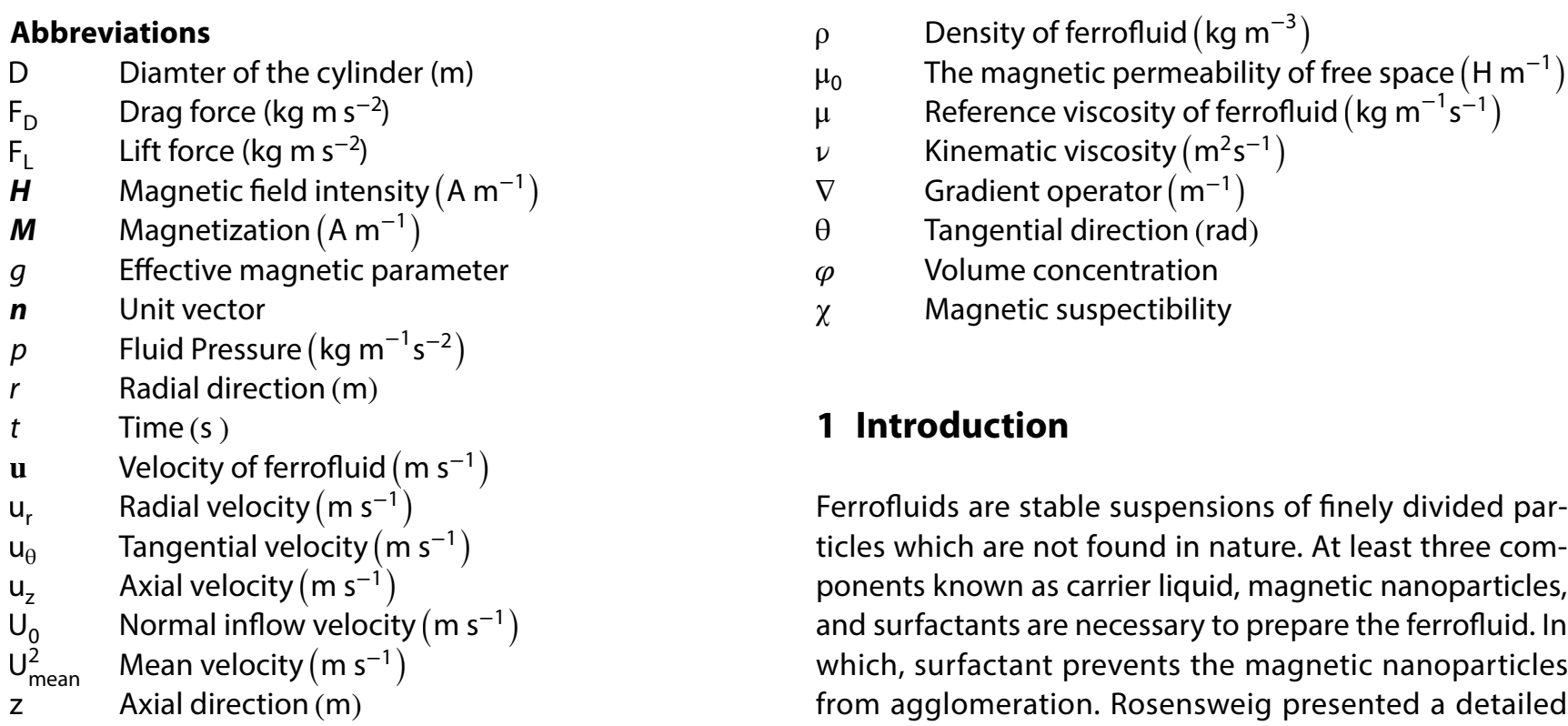

Anupam Bhandari, pankaj.anupam6@gmail.com, https://www.upes.ac.in | 1 Department of Mathematics, School of Engineering, University of Petroleum and Energy Studies (UPES), Energy Acres Building, Bidholi, Dehradun, Uttarakhand 248007, India. 
description of ferrohydrodynamics in his monograph [1]. Odenbach described the theoretical and experimental study on the viscosity the magnetic fluid in the presence/ absence of the magnetic field [2]. Shliomis validated the magnetization equations and described the rotational viscosity of ferrofluid [3]. Shliomis and Morozov explained that the viscosity of ferrofluid decreases under the influence of an alternating magnetic field in a certain frequency range [4]. Ferrofluids are important in the sealing of hard disc drive, electronically controllable signal, and improving the quality of loudspeakers [5]. One of the most important applications of ferrofluid is in the treatment of cancer through magetohyperthermia [6]. Odenbach and Thurm investigated about magnetic field dependent viscosity in the presence of the stationary and alternating magnetic field [7]. Bacari et al. described the experimental results for negative viscosity in the presence of an alternating magnetic field and compared it with the theoretical results [8].

Bognar and Hriczo studied water-based ferrofluid flow due to stretching and moving surfaces and considered the impact of suction and injection over the stretching sheet $[9,10]$. Moorthi and Sharma studied two-dimensional laminar flow in a no-circular channel using COMSOL Multiphysics [11]. Rosensweig et al. theoretically investigated the viscosity of a magnetic fluid in the presence of a magnetic field [12]. Siddheshwar et al. presented the concept of ferroconvection and electroconvection for rotating magnetic fluid [13]. However, the rotational flow of ferrofluid is useful in the sealing of a rotating shaft $[14,15]$. Bijarchi et al. investigated ferrofluid droplet manipulation under the influence of an alternating magnetic field for biomedical applications [16]. Favakeh et al. investigated the application of an alternating magnetic field in the elimination of satellite ferrofluid droplets [17]. Bijarchi et al. studied the formation of ferrofluid droplets from a nozzle under the influence of the non-uniform magnetic field at different angles [18]. Bijarchi and Shafii investigated experimentally the formation of ferrofluid droplets using a nozzle in the presence of a time-dependent nonuniform magnetic field utilizing a pulse-width-modulation signal [19]. Underwood investigated the incompressible flow past a circular cylinder for the Reynolds number up to ten [20]. Jiang investigated instantaneous and time-averaged flow past a circular cylinder in the subcritical regime [21]. Sierra et al. studied the bifurcation of two-dimensional laminar flow past a rotating cylinder for the Reynolds number up to two hundred [22]. Sooraj et al. investigated the effect of superhydrophobicity on the flow pas a cylinder through surface modifaction [23].

The present investigation describes the numerical study of two-dimensional time-dependent ferrofluid flow past a cylinder in the presence of 10 kilo-ampere per meter magnetic field. In the absence of a magnetic field, there is no impact of magnetic field dependent viscosity and magnetization force in the behavior of the flow. The flow of ferromagnetic fluid in the presence of a magnetic field is compared with the case in the absence of a magnetic field. The size of the magnetic nanoparticles is considered nine nanometers. Two-dimensional plots for velocity and pressure distributions are presented. The results for the drag coefficient and lift coefficient are also studied. The Reynolds number in the flow is about 100 and it is enough to keep the flow laminar. The higher values of Reynolds number in such types of flow represents some curious phenomena such as steady symmetric flow pattern of twin vortices, the origination of unsteady flow, Karman vortex street, and the progression from laminar flow to turbulent flow. A high Reynolds number also decreases the drag coefficient. In general, the Reynolds number is the ratio of inertial forces to viscous forces subjected to the internal movement due to different fluid velocities. The flow is incompressible for which the Mach number is greater than 0.3 . Mach number represents the ratio of flow velocity past a boundary to the local speed of sound.

\section{Mathematical formulation}

Figure 1 shows the domain of the flow region. The rectangular geometry is drawn first in COMSOL, which is $2.2 \mathrm{~m}$ in length and $0.4 \mathrm{~m}$ in breadth. Then a circle of radius $0.05 \mathrm{~m}$ at $(0.2,0.2)$ positions have been drawn. Then the difference is taken of these two geometries and then build all regions is selected. In the next step, the properties of ferrofluid are added under the section material properties.

The position of rectangle and circle are as follows:

Rectangle: Position (0, 0); Width (2.2); Height (0.4) and size $(2.2,0.4)$.

Circle: Position $(0.2,0.2)$; radius $(0.05)$.

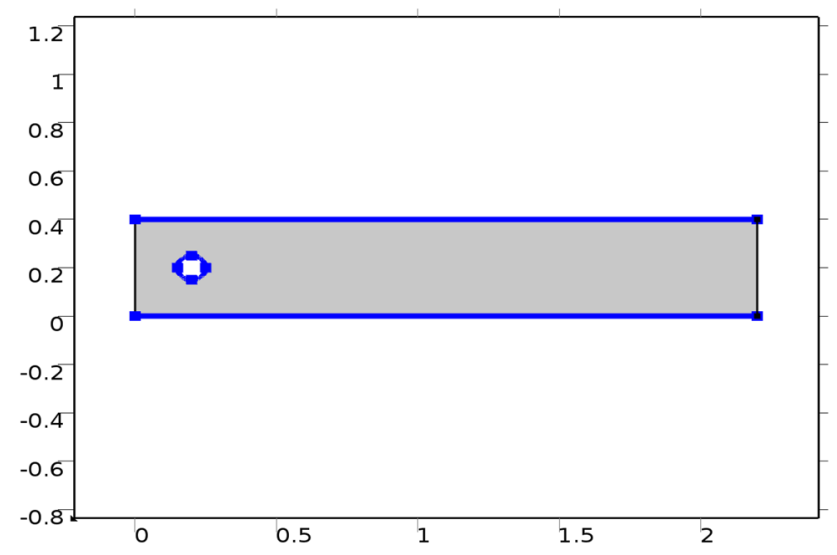

Fig. 1 Sketch of flow geometry for two-dimensional cylindrical flow 
The constitutive set of equations for ferrohydrodynamic flow is as follows[1, 4, 15]:

The equation of momentum

$\rho \frac{\partial \boldsymbol{u}}{\partial t}+\rho(\boldsymbol{u} \cdot \nabla) \boldsymbol{u}=-\nabla p+\mu_{0}(\boldsymbol{M} \cdot \nabla) \boldsymbol{H}+\mu\left(1+\frac{3}{2} \varphi g\right) \nabla^{2} \boldsymbol{u}$

The equation of continuity

$\nabla \cdot \boldsymbol{u}=0$

Maxwell Equations

$\nabla \times \boldsymbol{H}=0, \nabla \cdot(\boldsymbol{H}+\boldsymbol{M})=0$ with $\boldsymbol{M}=\chi \boldsymbol{H}$

Equations (1) and (2) can be reduced into two-dimensional cylindrical form are as follows:

$$
\begin{aligned}
& -\frac{1}{\rho} \frac{\partial \boldsymbol{p}}{\partial r}+v\left(1+\frac{3}{2} \varphi g\right)\left[\frac{\partial^{2} u_{r}}{\partial r^{2}}+\frac{\partial}{\partial r}\left(\frac{u_{r}}{r}\right)+\frac{\partial^{2} u_{r}}{\partial z^{2}}\right] \\
& +\mu_{0} M \frac{\partial H}{\partial r}=\left[\frac{\partial u_{r}}{\partial t}+u_{r} \frac{\partial u_{r}}{\partial r}+u_{z} \frac{\partial u_{r}}{\partial z}-\frac{u_{\theta}^{2}}{r}\right] \\
& v\left(1+\frac{3}{2} \varphi g\right)\left[\frac{\partial^{2} u_{\theta}}{\partial r^{2}}+\frac{\partial}{\partial r}\left(\frac{u_{\theta}}{r}\right)+\frac{\partial^{2} u_{\theta}}{\partial z^{2}}\right] \\
& =\left[\frac{\partial u_{\theta}}{\partial t}+u_{r} \frac{\partial u_{\theta}}{\partial r}+u_{z} \frac{\partial u_{\theta}}{\partial z}+\frac{u_{r} u_{\theta}}{r}\right] \\
& -\frac{1}{\rho} \frac{\partial \mathbf{p}}{\partial z}+v\left(1+\frac{3}{2} \varphi g\right)\left[\frac{\partial^{2} u_{z}}{\partial r^{2}}+\frac{1}{r} \frac{\partial u_{z}}{\partial r}+\frac{\partial^{2} u_{z}}{\partial z^{2}}\right] \\
& +\mu_{0} M \frac{\partial H}{\partial z}=\left[\frac{\partial u_{z}}{\partial t}+u_{r} \frac{\partial u_{z}}{\partial r}+v_{z} \frac{\partial u_{z}}{\partial z}\right] \\
& \frac{\partial u_{r}}{\partial r}+\frac{u_{r}}{r}+\frac{\partial u_{z}}{\partial z}=0
\end{aligned}
$$

In Eq. (1), in the presence of a magnetic field, the expression $\mu\left(1+\frac{3}{2} \varphi g\right)$ represent the magnetic field dependent viscosity. It creates additional resistance to the flow. In the absence of a magnetic field, there is no change in viscosity.
In Fig. 1, the velocity $\boldsymbol{u}$ is zero in the wall shown in blue lines. The boundary conditions are considered no slip and the initial pressure is taken zero. Then inlet and outlet conditions are selected in COMSOL. A magnetization force of 10-kilo ampere per meter is directed in the radial direction. For solving the Navier-Stokes equation with Maxwell equations, physics of the magnetic field with no current is added in COMSOL. The boundaries of the region of flow are shown in Fig. 1. The boundary conditions are as follows:

$$
\begin{aligned}
& \text { Region 'domain' } \\
& \text { Start'outer' }(0,0) \\
& \{\text { mirror conditions on bottom boundary }\} \\
& \text { Natural }\left(u_{r}\right)=0 \\
& \text { natural }\left(u_{\theta}\right)=0 \text { value }\left(u_{z}\right)=0 \\
& \text { natural }(\mathrm{p})=0 \text { line to }(0.4,0) \\
& \{\text { No slip on sides (i.e., velocity }=0)\} \\
& \text { value }\left(u_{r}\right)=0 \text { value }\left(u_{\theta}\right)=0 \text { value }\left(u_{z}\right)=0 \\
& \text { natural }(p)=0 \text { line to }(0.4,2.2) \\
& \text { natural }\left(u_{r}\right)=0 \text { natural }\left(u_{\theta}\right)=0 \\
& \text { value }\left(u_{z}\right)=0 \quad \text { value }(p)=0 \text { line to }(0,2.2) \\
& \text { Value }\left(u_{r}\right)=0 \text { value }\left(u_{\theta}\right)=0 \\
& \text { value }\left(u_{z}\right)=0 \quad \text { natural }(p)=0 \text { line to close } \\
& \text { at } t=0 ; \quad u_{r}=0, u_{\theta}=0, u_{z}=0, p=0 \\
& \text { at } r=0.4 ; \quad z=2.2, \quad \boldsymbol{u}=-U_{0} n
\end{aligned}
$$

The drag and lift coefficients are defined as:

$C_{D}=\frac{2 F_{D}}{\rho U_{\text {mean }}^{2} D^{\prime}}, \quad C_{L}=\frac{2 F_{L}}{\rho U_{\text {mean }}^{2} D}$

Table 1 shows the values of the physical parameters of ferrofluid during numerical computation.
Table 1 The physical parameters of ferrofluid for cylindrical flow

\begin{tabular}{lll}
\hline Name & Expression & Description \\
\hline Density & 1400 kg per meter & Density of ferrofluid \\
Viscosity & 0.035 Pas. second & The viscosity of ferrofluid without magnetic field \\
Rotational viscosity & 0.04375 Pas. second & Viscosity in the presence of magnetic field \\
Surface tension & $26 \mathrm{mN}$ per meter & Surface tension on ferrofluid \\
Temperature & $25^{\circ} \mathrm{C}$ & Temperature during flow \\
Size of the particle & $9 \mathrm{~nm}$ & Size of the particle in a carrier liquid \\
\hline
\end{tabular}


Table 2 Description of mesh statistics

\begin{tabular}{ll}
\hline Property & Value \\
\hline Minimum element quality & 0.3395 \\
Average element quality & 0.9048 \\
Triangular elements & 25,314 \\
Quadrilateral elements & 1812 \\
Edge elements & 990 \\
Vertex elements & 8 \\
\hline
\end{tabular}

Table 3 Description of mesh size

\begin{tabular}{ll}
\hline Geometric entity level & Boundary \\
\hline Selection & Boundaries 2-3, 5-8 \\
Calibrate for & Fluid dynamics \\
Maximum element size & 0.0052 \\
Minimum element size & $6.0 \mathrm{E}-5$ \\
Curvature factor & 0.25 \\
The maximum element growth rate & 1.08 \\
Predefined size & Extra fine \\
\hline
\end{tabular}

\section{Procedure of numerical solution}

Physics controlled finer mesh is used in the solution. The total triangular elements are 25,314. Quadrilateral elements are 1812, edge elements are 990, and vertex elements are 8 . The maximum element size is taken 0.014 and the minimum element size is taken is 0.0004 . Mesh statistics is configured as:

In domain 1, the corner refinement option is selected in COMSOL. Sharp corners are handled through trimming options. This model is studied under the laminar flow section with a stationary magnetic field. Table 2 represents the description of mesh statistics. However, Table 3 represents the description of the mesh size. Extra fine mesh is used in the numerical solution. Even at the element size 0.018 , the solution is independent of the grid.

\section{Results and discussions}

Present results show the laminar flow of ferrofluid in cylindrical geometry. 2D Flow has been modeled here in the presence of an applied magnetic field 10 kiloampere/meter in the $z$-direction. In the present twodimensional plots, the vertical axis in the flow represents the diameter of the cylinder and the horizontal axis represents the $z$ direction. The fluid is considered

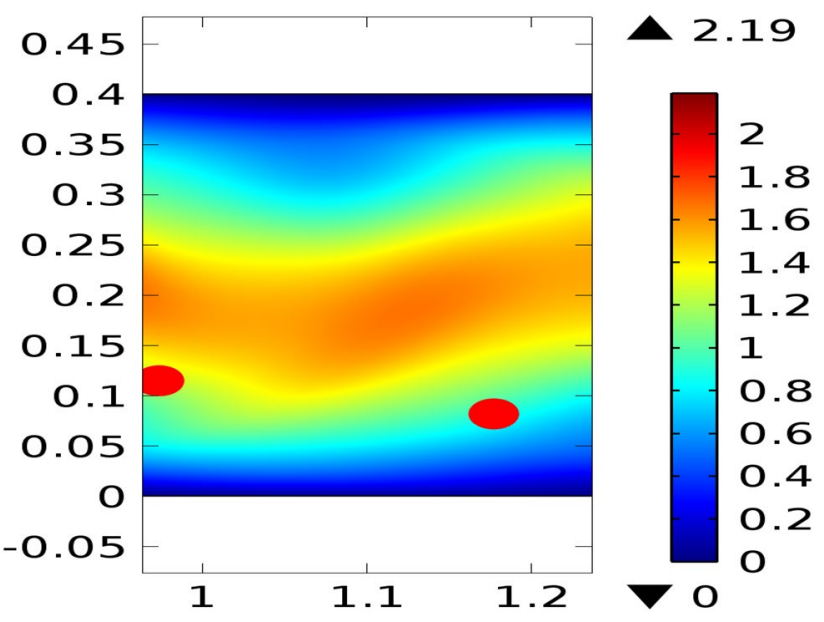

Fig. 2 Velocity distribution in two-dimensional cylindrical flow without magnetic field at $\mathrm{t}=7 \mathrm{~s}$

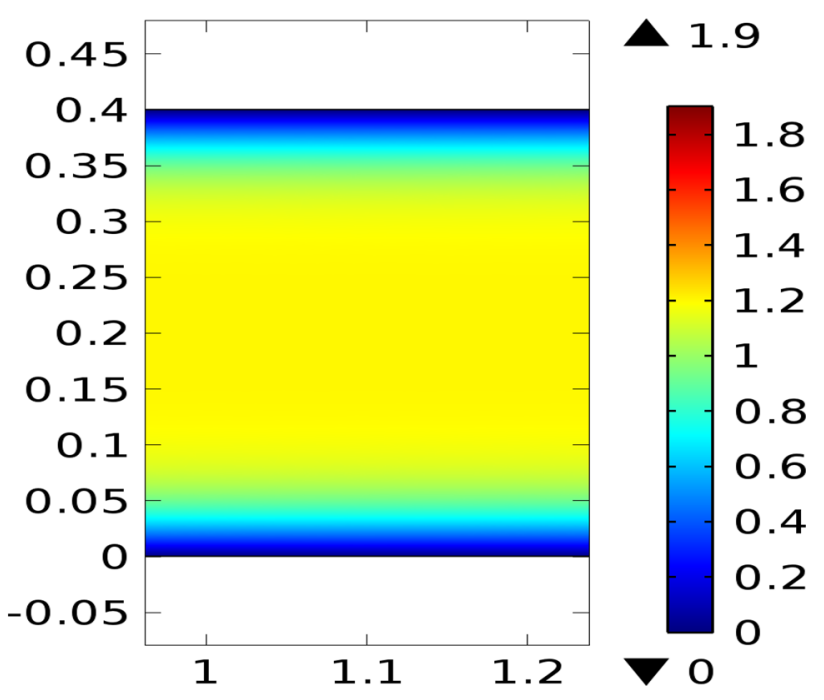

Fig. 3 Velocity distribution in two-dimensional cylindrical flow for $\mathrm{H}=10$ kilo-ampere per meter at $\mathrm{t}=4 \mathrm{~s}$

incompressible and time-dependent. Figure 2 depicts the velocity distribution without any magnetic field at $t=7 \mathrm{~s}$. In the absence of the magnetic field, the maximum value of the velocity is $2.19 \mathrm{~m} / \mathrm{second}$. Figures 3 and 4 represent the velocity distributions under the influence of 10 kilo-ampere per meter of magnetic field intensity at $\mathrm{t}=4$ and $7 \mathrm{~s}$, respectively. In the absence of a magnetic field, there is no impact of magnetic field dependent viscosity in the flow. However, the stationary magnetic field creates an additional resistance in the flow due to the misalignment of magnetic and viscous torques. As the fluid comes under the influence of the external magnetic field, the velocity decreases since the magnetic field creates an additional resistance on the 


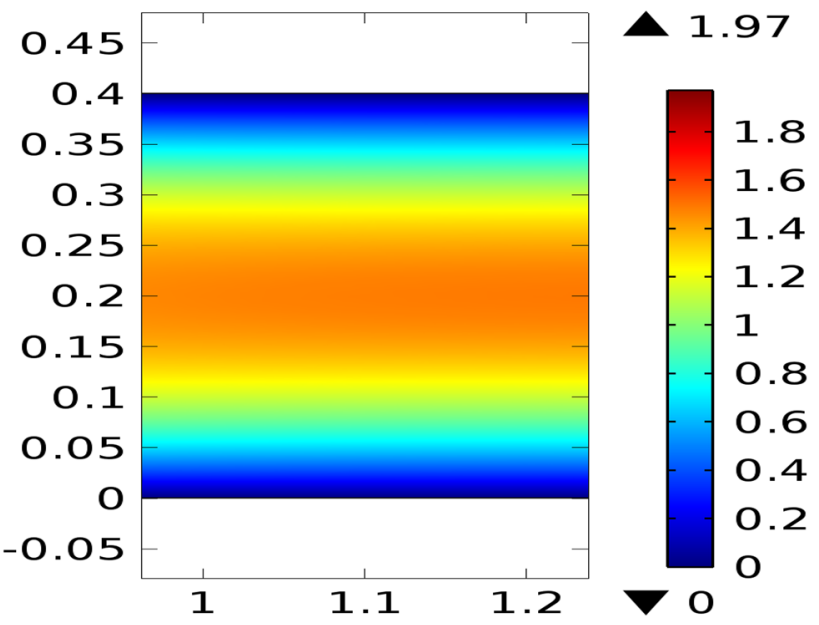

Fig. 4 Velocity distribution in two-dimensional cylindrical flow for $\mathrm{H}=10$ kilo-ampere per meter at $\mathrm{t}=7 \mathrm{~s}$

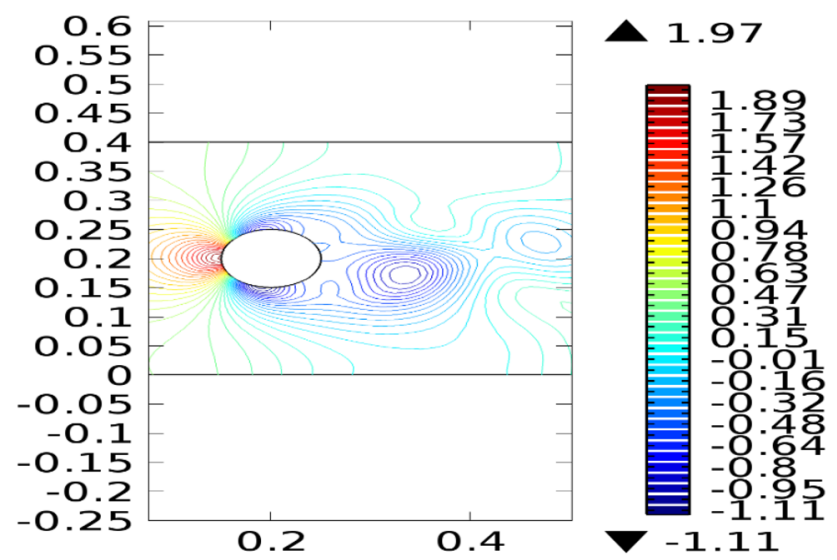

Fig. 5 Pressure distribution in two-dimensional cylindrical flow without magnetic field at $\mathrm{t}=7 \mathrm{~s}$

flow in a form of viscosity. The main physical reason for the magnetic field dependent viscosity in the flow is that the magnetic torque creates a difference between the velocity of a fluid and nanoparticles. Therefore, the difference between the rotation of magnetic fluid and nanoparticles reduces the velocity distribution in the flow. The role of the magnetization body force and density is also an important factor in the velocity reduction. The present results indicate that the velocity distributions does not depend only on the strength of the magnetic field but also on the time. In the absence of the magnetic field, the maximum velocity of the fluid is in the small diameter of the cylinder. However, in the presence of a magnetic field, it is in the wider region as compared to the previous case.

Figure 5 shows the pressure distribution in the fluid without any magnetic field. However, Figs. 6 and 7 show

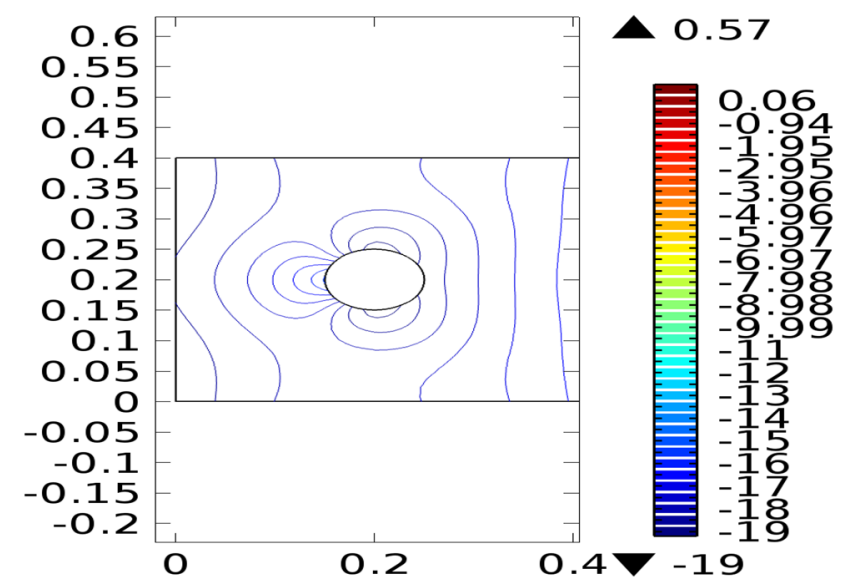

Fig. 6 Pressure distribution in two-dimensional cylindrical flow for $\mathrm{H}=10$ kilo-ampere per meter at $\mathrm{t}=4 \mathrm{~s}$

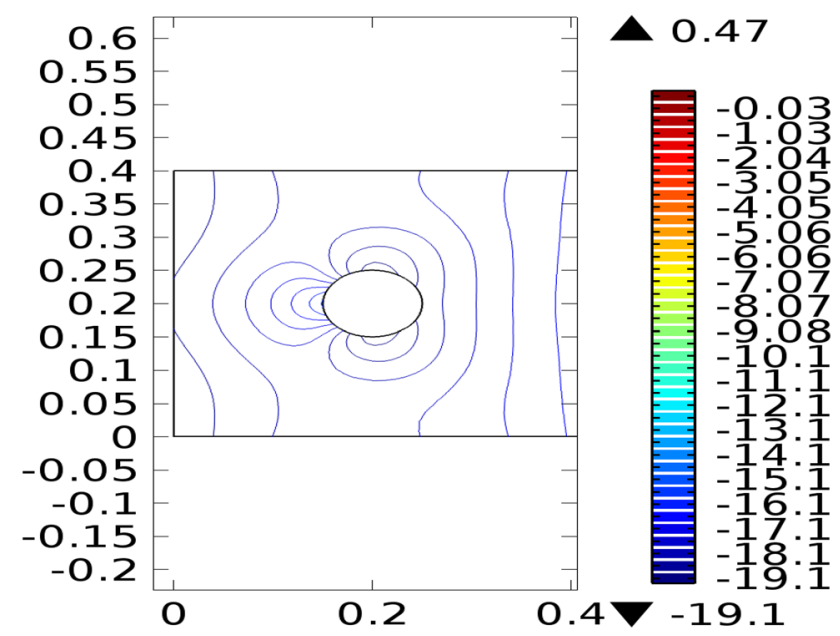

Fig. 7 Pressure distribution in two-dimensional cylindrical flow for $\mathrm{H}=10$ kilo-ampere per meter at $\mathrm{t}=7 \mathrm{~s}$

the pressure distribution in presence of a magnetic field of strength 10 kilo-ampere per meter. In the momentum equations, the pressure force and magnetization force have opposite signs. The expressions in Eqs. (4) and (6) indicate that these forces are working in opposite directions. In the absence of a magnetic field, the magnitude of the pressure is 1.97. However, the magnetization force reduces the pressure distribution in the flow. However, in the presence of a magnetic field, the magnitude of the pressure in the flow is higher than without a magnetic field. Two-dimensional present plots show that the magnetization force is dominant over the pressure force in the flow. The negative values of the pressure show that the magnetic field intensifies the pressure in opposite direction. The main impact of the pressure is in front of the circular wall and the backside of the circular region is negligible. The magnetization force acts on 
the fluid throughout the volume; therefore, it reduces the pressure distribution in the flow.

Figures 8 and 9 depict the distribution of the drag and lift coefficients. In the presence of a magnetic field, the drag coefficient in the flow higher than without the magnetic field since the average velocity in the flow decreases. Fluctuations are quite higher in the drag coefficient of ferrofluid under the influence of the external magnetic field. The lift coefficient shown in Fig. $9 \mathrm{~b}$ is higher as compared to the presented in Fig. 9a. This force act on the perpendicular direction in the flow, therefore, it increases sharply in the presence of a magnetic field. The average velocity of the magnetic fluid plays an important role in the variation of the drag and lift coefficients. The averaged velocity is reduced therefore the drag and lift coefficients are higher in the presence of a magnetic field. The drag and lift coefficient is very important to understand the characteristics of the fluid.

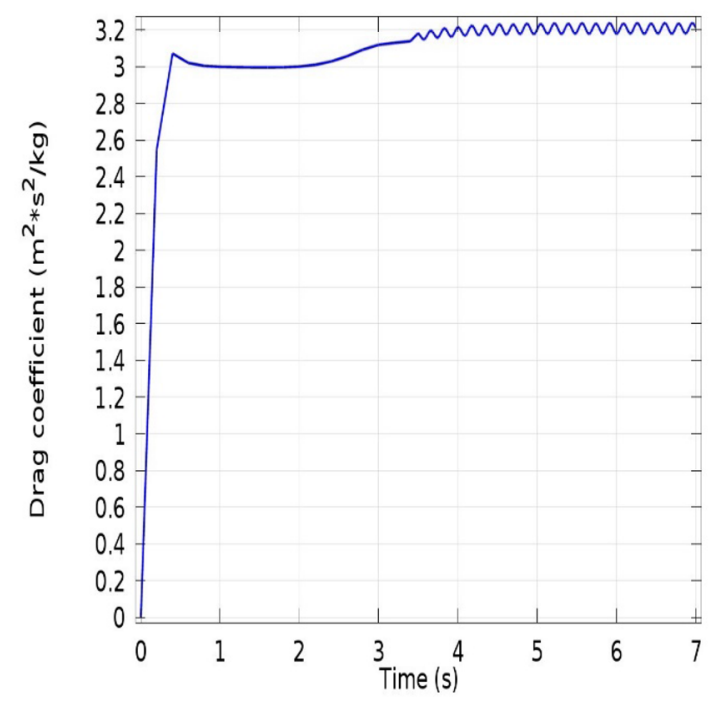

(a)

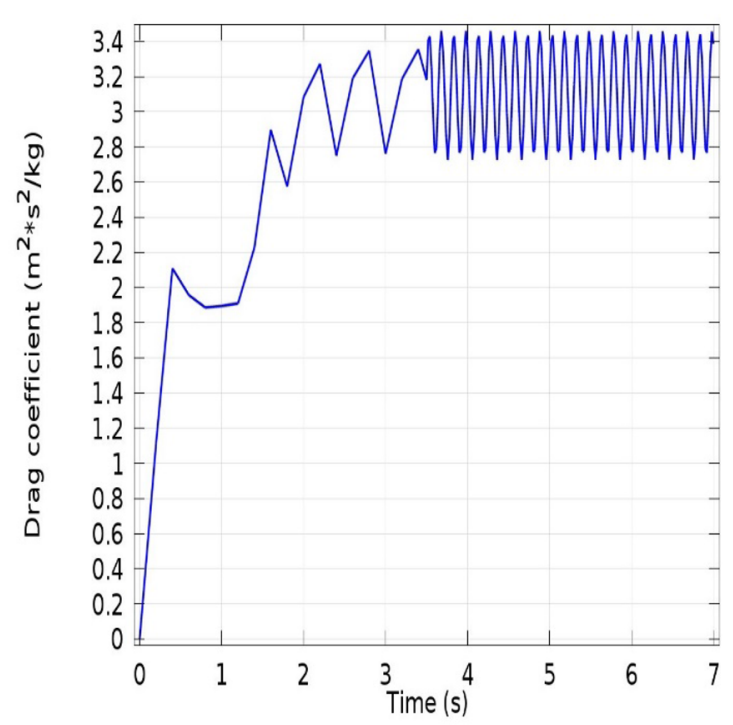

(b)

Fig. 8 a Drag coefficient without magnetic field. $\mathbf{b}$ Drag coefficient at $\mathrm{H}=10$ kilo-ampere/meter

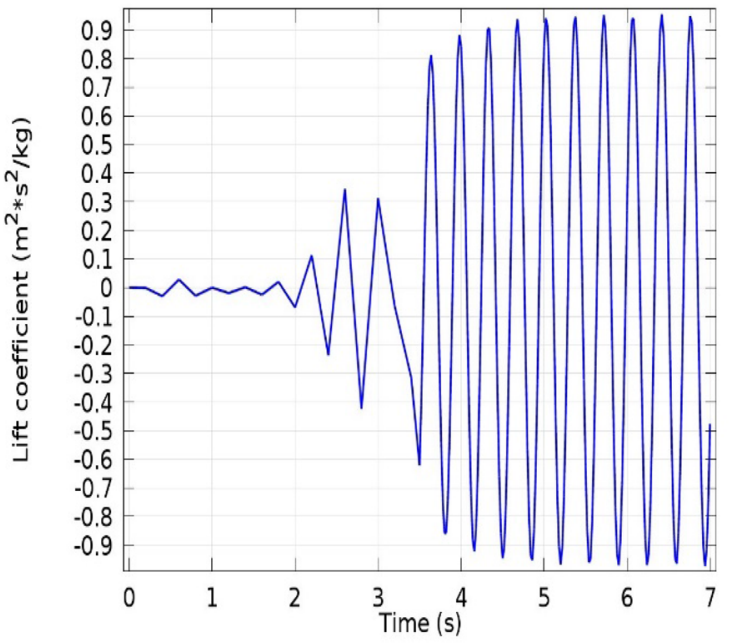

(a)

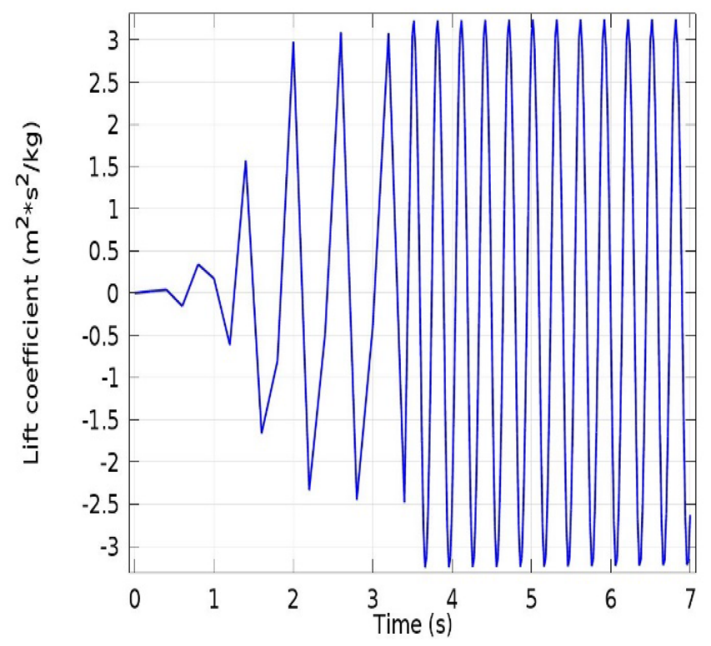

(b)

Fig. 9 a Lift coefficient without magnetic field. $\mathbf{b}$ Lift coefficient at $\mathrm{H}=10$ kilo-ampere/meter 


\section{Conclusions}

Two-dimensional time-dependent ferrofluid flow pas in a cylinder is studied in the presence of a stationary magnetic field and compared the study in the absence of a magnetic field. In the presence of a magnetic field, the velocity in the flow reduces due to magnetic field dependent viscosity and magnetization force. The reduction in the viscosity enhances the drag and lift coefficients in the flow. This type of stimulation may play an important role in drug targeting during the magetohyperthermia in the treatment of liver cancer through the magnetic fluid. The present study can be extended for the microchannel flow of magnetic fluid for the possible applications in the medical field. The main results of the present study are as follows:

- In the presence of 10 kilo-ampere per meter magnetic field, the velocity drops from 2.19 to $1.97 \mathrm{~m} / \mathrm{s}$ at $\mathrm{t}=7 \mathrm{~s}$.

- At the same intensity of magnetic field and time, the lift coefficients enhance from 3 to $3.4 \mathrm{~m}^{2} \mathrm{~s}^{2} / \mathrm{kg}$ and the drag coefficient enhances from $0.9 \mathrm{~m}^{2} \mathrm{~s}^{2} / \mathrm{kg}$ to $3 \mathrm{~m}^{2} \mathrm{~s}^{2} /$ $\mathrm{kg}$.

- The magnetic field reduces the pressure in front of the circular region in the flow.

\section{Compliance with ethical standards}

Conflict of interest The author declares that he has no conflict of interest.

Open Access This article is licensed under a Creative Commons Attribution 4.0 International License, which permits use, sharing, adaptation, distribution and reproduction in any medium or format, as long as you give appropriate credit to the original author(s) and the source, provide a link to the Creative Commons licence, and indicate if changes were made. The images or other third party material in this article are included in the article's Creative Commons licence, unless indicated otherwise in a credit line to the material. If material is not included in the article's Creative Commons licence and your intended use is not permitted by statutory regulation or exceeds the permitted use, you will need to obtain permission directly from the copyright holder. To view a copy of this licence, visit http://creativecommons .org/licenses/by/4.0/.

\section{References}

1. Rosensweig RE (1985) Ferrohydrodynamics. Cambridge University Press, Cambridge

2. Odenbach $S$ (2000) Magnetoviscous effects in ferrofluids. Appl Rheol 10(4):178-184

3. Shliomis MI (2001) Ferrohydrodynamics: testing a third magnetization equation. Phys Rev E 64(6):060501
4. Shliomis MI, Morozov KI (1994) Negative viscosity of ferrofluid under alternating magnetic field. Phys Fluids 6(8):2855-2861

5. Blums E, Cebers A, Maiorov M (2010) Magnetic fluids. Walter de Gruyter, Berlin

6. Malaescu I, Fannin PC, Marin CN, Lazic D (2018) The concept of ferrofluid preheating in the treatment of cancer by magnetic hyperthermia of tissues. Med Hypotheses 110:76-79

7. Odenbach S (2002) Ferrofluids: magnetically controllable liquids. PAMM 1(1):28

8. Bacri JC, Perzynski R, Shliomis MI, Burde GI (1995) Negative-viscosity effect in a magnetic fluid. Phys Rev Lett 75(11):2128-2131

9. Bognár G, Hriczó K (2020) Numerical simulation of water based ferrofluid flows along moving surfaces. Processes 8(7):830

10. Bognár G, Hriczó K (2020) Ferrofluid flow in magnetic field above stretching sheet with suction and injection. Math Model Anal 25(3):461-472

11. Moorthi A, Sharma AK (2018) Laminar fluid flow and heat transfer in non-circular sub-channel geometries of nuclear fuel bundle. Prog Nucl Energy 103:243-253

12. Rosensweig RE, Kaiser R, Miskolczy G (1969) Viscosity of magnetic fluid in a magnetic field. J Colloid Interface Sci 29(4):680-686

13. Siddheshwar PG, Suthar OP, Chinnaswamy K (2019) Finite-amplitude ferro-convection and electro-convection in a rotating fluid. SN Appl Sci 1(12):1-11

14. Bhandari A (2020) Study of ferrofluid flow in a rotating system through mathematical modeling. Math Comput Simul 178:290-306

15. Bhandari A (2020) Study of magnetoviscous effects on ferrofluid flow. Eur Phys J Plus 135(7):537

16. Bijarchi MA, Favakeh A, Sedighi E, Shafii MB (2020) Ferrofluid droplet manipulation using an adjustable alternating magnetic field. Sens Actuators A Phys 301:111753

17. Favakeh A, Bijarchi MA, Shafii MB (2020) Ferrofluid droplet formation from a nozzle using alternating magnetic field with different magnetic coil positions. J Magn Magn Mater 498:166134

18. Bijarchi MA, Favakeh A, Shafii MB (2020) The effect of a nonuniform pulse-width modulated magnetic field with different angles on the swinging ferrofluid droplet formation. J Ind Eng Chem 84:106-119

19. Bijarchi MA, Shafii MB (2020) Experimental investigation on the dynamics of on-demand ferrofluid drop formation under a pulse-width-modulated nonuniform magnetic field. Langmuir 36(26):7724-7740

20. Underwood RL (1969) Calculation of incompressible flow past a circular cylinder at moderate Reynolds numbers. J Fluid Mech 37(1):95-114

21. Jiang $H$ (2020) Separation angle for flow past a circular cylinder in the subcritical regime. Phys Fluids 32(1):014106

22. Sierra J, Fabre D, Citro V, Giannetti F (2020) Bifurcation scenario in the two-dimensional laminar flow past a rotating cylinder. $J$ Fluid Mech 905:A2. https://doi.org/10.1017/jfm.2020.692

23. Sooraj P, Ramagya MS, Khan MH, Sharma A, Agrawal A (2020) Effect of superhydrophobicity on the flow past a circular cylinder in various flow regimes. J Fluid Mech 897:A21. https://doi. org/10.1017/jfm.2020.371

Publisher's Note Springer Nature remains neutral with regard to jurisdictional claims in published maps and institutional affiliations. 